\title{
Microstructure Based Failure Criterion For Ductile Materials
}

\author{
Saryu Fensin ${ }^{1, *}$, George Gray III $^{1}$, Neil Bourne ${ }^{2}$, and Robert Hixson ${ }^{1}$ \\ ${ }^{1}$ Materials Science and Technology Division, Los Alamos National Laboratory, Los Alamos, USA 87544 \\ ${ }^{2}$ University of Manchester, Rutherford Appleton Laboratory, Didcot, Oxfordshire, OX11 0DE, United Kingdom
}

\begin{abstract}
For ductile metals, the process of dynamic fracture occurs through nucleation, growth and coalescence of voids. The stress required to nucleate these voids is inferred from the velocimetry data (using the acoustic approach) and termed as the spall strength. This is a key parameter that is used to evaluate a material's susceptibility to damage and failure. However, it is also well recognized that the dynamic parameters used to generate the shock state such as pulse duration, tensile strain-rate and peak stress coupled with material microstructure itself affect the material response in a complex manner. Yet, it is impossible to capture all this information by assessing only the spall strength measured from simple one-dimensional Photon Doppler Velocimetry measurements. Although, there exist widely used corrections proposed by Kanel et. al. that allow for the inclusion of some of these complexities into the measured spall strength but still does not take the microstructure into account. In this work, we propose another scheme for normalization of spall strength with a damage area to capture the complexities included in the damage and failure process especially pertaining to microstructure. We will also demonstrate the application of this scheme by applying to examples of materials such as Copper, Copper-24 wt\% Ag, Copper-15 wt $\%$ Nb and additively manufactured 316L SS.
\end{abstract}

\section{Introduction}

Multiple notions of strength may be applied to a given material depending on the loading conditions. A few related to uniaxial loading include yield and ultimate strengths. Under dynamic loading conditions, one type of strength related to material failure is frequently termed "spall or dynamic tensile strength". The tensile dynamic strength is one of the key properties that is used to assess the materials resistance to damage nucleation under high strain rates [1]. The simplest way to generate dynamic loading conditions in a material involves high velocity impact with a flyer. This gives rise to a compression or shock wave, of a given amplitude, in the material and the flyer. These shock waves then get reflected from the free surfaces in both the flyer and the material. The generated release waves travel through the flyer and material until they intersect at a predetermined location. The material is then driven into tension in the specific region of intersection leading to the creation of damage in the form of voids in ductile materials, which grow and coalesce finally leading to failure under sufficiently large tension. Parameters such as the peak stress, pulse duration, pulse shape, tensile strain-rate associated with the shock wave can all be controlled to some extent by altering either the flyer thickness and material or using high explosives or lasers to generate shock in the material $[2,3]$. These loading parameters have a profound effect on the type and amount of damage generated in the material. In general, these loading parameters have complex interactions with the microstructure of a material

\footnotetext{
*e-mail: saryuj@lanl.gov
}

especially if they have elastic-plastic behavior. For example, for a given microstructure altering the pulse duration of the shock wave will not only alter the total deformation in the material under compression but this will also alter the dynamic strength of the material $[4,5]$. This change is tied to the fact that deformation in the form of dislocations is an important stress dissipation mechanism[6]. These types of affects cannot be simply accounted for by measuring only the spall strength of the material. The total deformation in the material not only affects the nucleation stress for voids but will also alter the growth and coalescence of these voids. Spall strength will only provide information regarding the stress required to open enough "free surfaces" within the sample such that the stress wave gets trapped between the spall scab and the free surface causing a change in the velocity time history being measured on the surface but no information regarding the total damage in the material [1]. Hence, to capture the effect of microstructure on the total damage in the material, for the purpose of developing microstructurally-aware predictive models for spallation, it is indispensable to include quantification of both the damage mechanism as well as the kinetics and volume within the sample over which applied energy is dissipated via evolved damage in addition to spall strength.

Experiments to probe the dynamic strength of materials generally measure spall strength from the free surface velocity history $\left(\mathrm{u}_{f s}(t)\right)$ via photonics doppler velocimetry (PDV) or VISAR, which is recorded at the surface of the material instead of inside the material where spall nucleates. It is well established that there are issues with 
these measurements associated with the transmission of information from the bulk to the free surface due to the elastic-plastic nature of most ductile materials. Multiple ways of accounting for errors in these measurements have been discussed by Kanel et. al.[1] in the form of corrections that are made to the measured spall strength. In general, spall strength is calculated by the simple relationship [7]:

$$
\sigma_{s p}=\frac{1}{2} \rho_{o} c_{o} \Delta u_{f s}
$$

where $\rho_{o}$ is the ambient density, $\mathrm{C}_{o}$ is the bulk sound velocity and $\Delta u_{f s}$ is the difference in the free surface velocity from the peak state to the pull-back minima. This assumes linear compression of the material along with a constant sound speed. However, these assumptions fail in the limit of large spall strength values and other corrections need to made. The most applied corrections are the ones by Romanchenko et. al [8] and Kanel et. al [9] that attempt to take into account not the elastic-plastic nature of ductile materials but account for the interaction between the elastic and plastic compression waves. However, all these corrections are based on the transmission of acoustic waves within the sample and do not take any microstructural information into account. This information is important because previous work has shown that voids nucleate preferentially at pre-existing flaws like dislocations, grain boundaries, and inclusions in a given material $[2,3,10-15]$. But in addition to nucleation it has been shown the microstructure affects void growth and coalescence, which together comprise the total damage in the material $[4,16,17]$. Hence, just using the spall strength to make conclusions regarding the resistance of a material to damage misses the complete picture.

In this work, we propose to couple spall strength with the area or volume fraction of damage to indirectly include microstructural effects in this important failure criterion. The rest of the paper is organized as follows. The next section discusses the details and the rationale behind the proposed failure criterion and experimental details associated with the examples used to demonstrate the robustness of this criterion. Section 3 applies the criterion to a few problems within the material science community and we conclude in Section 4.

\section{Methodology}

This section outlines the proposed microstructure based failure criterion along with the experimental details associated with the examples discussed in the results section.

\subsection{New Failure Criterion}

The "spallation response" of a material can be quantified by both: 1) the measurement of the energy necessary to cause incipient or full spall scab formation as quantified via rear-surface PDV or VISAR wave profile "pull-back" signals, and 2) post-mortem analysis of the damage evolution in the sample following spall loading.
The importance of coupling the in-situ wave-profile signal with the damage evolution is to both assess the operative damage processes (such as ductile void formation, shear localization, brittle cracking, intergranular fracture) as well as the volume of the sample over which damage manifests. The development of microstructurally-aware predictive models for spallation is predicated on quantification of both the damage mechanism as well as the kinetics and volume within the sample over which this energy is dissipated.

Analogous to quantification of the "ductility" and damage evolution in a quasi-static tensile test is the importance of not only measuring the tensile strength (akin to measuring the spall strength) but also quantifying the "ductility" of the material. For the latter two measurements are done; the percent elongation - the ratio of the increase in length of the gage section of the sample to its original length, versus the second; the reduction in area defined as the percentage reduction of area of the decrease in the cross-sectional area of the tensile specimen. A high reduction of area indicates the ability of the metal to deform extensively without fracture or failure. Accordingly, the reduction of area is the most structure sensitive parameter that is measured in a tensile test. It is also the the most important aspect used to assess material strength and quality. A decrease in reduction in area from a specified level will act as a warning sign that the quality of the material is substandard. One material might display a large elongation yet fail in a brittle manner versus another material may exhibit limited tensile ductility yet locally neck to a point, i.e., a reduction in area of nearly $100 \%$ [18].

Transferring these two concepts to that of post-mortem spallation analysis can be fruitful. One can postulate two materials that exhibit similar or nearly identical spall pullback signals but very different damage behavior. One of the materials displays a narrow region of evolved damage leading to a fully separated spall scab versus the other material displays a broad region of nucleated and evolved damage only resulting in incipient damage evolved. Modeling of these two different materials would require radically different physics in the kinetics of spall nucleation and growth. Our proposed failure criterion seeks to advance this gap in knowledge with additional insights into damage evolution.

The proposed failure criterion takes into account the effect of volume or area over which damage is nucleated to assess its susceptibility to damage:

$$
\sigma_{s p}^{m i c r o}=\frac{\sigma_{s p}}{A F r a c_{D a m}},
$$

where $\sigma_{s p}$ is the spall strength measured traditionally from the PDV data and AFrac ${ }_{\text {Dam }}$ is the damage area fraction assessed from the metallography on the $2 \mathrm{D}$ crosssection of the recovered sample. Ideally, the damage area fraction would actually be a damage volume fraction but in this work we focus on normalizing by the area fraction since that can be assessed from a $2 \mathrm{D}$ cross-section of the sample whereas a volume fraction estimate requires serial sectioning of the sample. This will be included in the next 
version of the criterion but we do not expect it to change the trends in the resistance to damage.

\subsection{Experiments}

For all the examples discussed in Section 3, all targets were prepared with press-fit momentum trapping rings to mitigate perturbations from edge release waves. A typical square wave profile was achieved using a varying flyer materials at varying pressures. The details of which can be found in references describing the details of each study $[5,16,17]$. Impact velocities were measured to an accuracy of $1 \%$ using a sequential pressure transducer technique and sample tilt was fixed to $1 \mathrm{mrad}$ by means of an adjustable specimen mounting fixture. Following impact, all samples were soft recovered by decelerating them into low-density foam. The free surface velocity history $\left(\mathrm{u}_{f s}(t)\right)$ profiles were measured using multiple PDV probes and data was analyzed using the procedures described by Jensen et al [19]

Subsequent characterization of the damage in the spalled samples included optical and Electron Back Scatter Diffraction (EBSD) microscopy. In preparation for the optical and EBSD analyses, each recovered specimen was diametrically sectioned. They were subsequently mounted in an epoxy resin and prepared following standard metallographic techniques as listed in the references $[5,16,17]$.ImageJ analysis was performed on optical micrographs to quantify the total damage. As part of this analysis, images were obtained of the sample 2D cross section at 10x magnification. Each image was then converted to greyscale and then a threshold applied so that the voids were selected but other dark features such as grain boundaries were not. The selected voids were then subjected to a particle size analysis to provide data such as number of particles and area percentage.

\section{Results}

\subsection{Example 1: Grain Size Effects in Copper}

Work by Escobedo et. al investigated four different grain size ranging from 30-200 $\mu \mathrm{m}$ in copper to study the effect of grain size on the spall strength and total nucleated damage within the samples [17]. The results from this study showed that the measured spall strength was independent of grain size. In fact, the PDV profiles were found to be identical for each study except for the slope of the pullback signals. If spall strength is simply the stress required to nucleate a void within a sample and the distribution of the weak links in form of grain boundaries in the different samples was unchanged then these results would be valid. However, postmortem analysis of these materials showed that the total damage in the recovered sample varied as a function of grain size, which is inconsistent with the conclusions solely derived from the PDV profiles. This work highlights the importance of sample recovery in experiments focusing on understanding the role of microstructure on damage and failure. The new failure criterion applied to the data reported in the paper to test the robustness of this new criterion and gain insights into the effect of microstructure on damage and failure as shown in Table 1.

Table 1. Spall strength, damage fraction and the normalized failure criterion for Copper.

\begin{tabular}{cccc}
\hline Material & $\begin{array}{c}\text { Spall Strength } \\
\text { GPa }\end{array}$ & $\begin{array}{c}\text { Damage Area Fraction } \\
\text { Unitless }\end{array}$ & $\begin{array}{c}\text { Failure Criterion } \\
\text { GPa }\end{array}$ \\
\hline $\mathrm{Cu}-30 \mu m$ & 1.38 & 0.496 & 2.78 \\
$\mathrm{Cu}-60 \mu m$ & 1.36 & 0.249 & 5.46 \\
$\mathrm{Cu}-100 \mu \mathrm{m}$ & 1.31 & 0.419 & 3.13 \\
$\mathrm{Cu}-200 \mu \mathrm{m}$ & 1.38 & 0.507 & 2.72 \\
\hline
\end{tabular}

The new failure criterion indeed shows that the largest grain size material was the weakest, followed by the 30 $\mu m, 100 \mu m$ and finally the $60 \mu m$ material. This conclusion was also reached in the initial study but through a lengthy discussion, which involved separate discussion of the spall strength and the measured damage. The new failure criterion not only simplifies this analysis but can be used as a failure criterion, which could reproduce the experimentally observed results. Using the original spall strength as the only failure criterion would have not captured the varying amounts of damage observed in the samples. Until we have microstructure based models that can evolve the nucleated damage, based on microstructural features, this new criterion can be used indirectly capture the total strength of the material under dynamic loading conditions.

\subsection{Example 2: $\mathrm{Cu}, \mathrm{Cu}-24 \mathrm{wt} \% \mathrm{Ag}$ and $\mathrm{Cu}-10 \mathrm{wt} \% \mathrm{Nb}$}

The question of strength in materials becomes even more complex for multi-component alloys.

Table 2. Spall strength, damage area fraction and the normalized failure criterion for $\mathrm{Cu}, \mathrm{CuAg}$ and $\mathrm{CuNb}$.

\begin{tabular}{cccc}
\hline Material & $\begin{array}{c}\text { Spall Strength } \\
\text { GPa }\end{array}$ & $\begin{array}{c}\text { Damage Area Fraction } \\
\text { Unitless }\end{array}$ & $\begin{array}{c}\text { Failure Criterion } \\
\text { GPa }\end{array}$ \\
\hline $\mathrm{Cu}$ & 1.328 & 0.967 & 1.37 \\
$\mathrm{CuAg}$ & 1.421 & 1.826 & 0.77 \\
$\mathrm{CuNb}$ & 1.806 & 1.045 & 1.73 \\
\hline
\end{tabular}

In this section, we discuss the effect of adding Ag and $\mathrm{Nb}$ to copper on its susceptibility to damage [5]. The details of the experiments are listed in Ref. [5] In the case of $\mathrm{Cu}$ alloyed with $\mathrm{Ag}$ and $\mathrm{Nb}$ simply considering the spall strength measured from the PDV profiles would suggest that $\mathrm{Cu}$ nucleated the most amount of damage followed by $\mathrm{CuAg}$ and $\mathrm{CuNb}$ as listed in Table 2. However, the total amount of damage present in the samples (as shown in Fig. 1) is not consistent with this conclusion.

Normalization of the spall strength with the damage area fraction alters the results to show that $\mathrm{CuAg}$ is the weakest material followed by $\mathrm{Cu}$ and then $\mathrm{CuNb}$. This is not only consistent with the quantitative results but also logically in terms of "why" that should be the case from the observed damage evolution. 


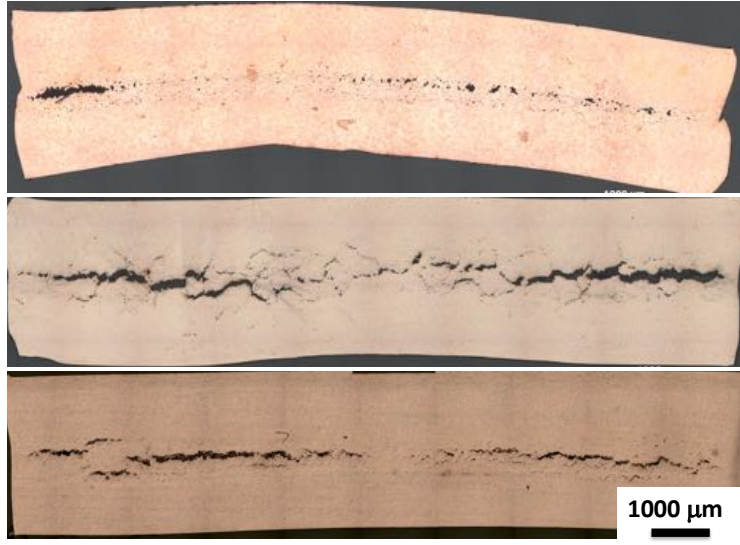

Fig. 1. Optical micrographs of Copper (Top), $\mathrm{Cu}-\mathrm{Ag}$ (middle) and $\mathrm{Cu}-\mathrm{Nb}$ (bottom). The larger black regions represent voids.

\subsection{Example 3: Wrought, additively manufactured, additively manufactured and heat treated $316 \mathrm{~L}$ Stainless steel}

Processing can be used to alter the microstructure of materials and additive manufacturing $(\mathrm{AM})$ is one such process. Experiments were performed to investigate the dynamic strength of additively manufactures 316L SS in comparison with its wrought counterpart the details of which are discussed in Ref. [16]. Additional heat treatments were

Table 3. Spall strength, damage area fraction and the normalized failure criterion for $316 \mathrm{~L} \mathrm{SS}$.

\begin{tabular}{cccc}
\hline Material & $\begin{array}{c}\text { Spall Strength } \\
\text { GPa }\end{array}$ & $\begin{array}{c}\text { Damage Area Fraction } \\
\text { Unitless }\end{array}$ & $\begin{array}{c}\text { Failure Criterion } \\
\text { GPa }\end{array}$ \\
\hline Wrought & 2.57 & 1.86 & 1.38 \\
AM-RX & 2.87 & 1.569 & 1.83 \\
AM & 3.43 & 0.983 & 3.49 \\
\hline
\end{tabular}

performed on the additive material to induce recrystallization in order to erase the processing history of the material. The spall strength measurements suggested the material with the strongest resistance to damage to be the $316 \mathrm{~L}$ AM followed by AM heat treated material (AM-RX) and then the 316L SS wrought, respectively as shown in Table 3 This observation is consistent with the total amount of damage shown in Fig. 2. However, without the postmortem analysis, the pull-back data would lead to spurious conclusions. In this specific case, the new failure criterion actually matches the conclusions drawn from the spall strength trends. However, without the recovery analysis it would have been impossible to observe the differences in the morphology of the nucleated voids in the AM material. This is a case where even though the resistance to damage information in the material is not altered by our analysis but it stresses the importance of recovery when trying to infer the effect of microstructure on damage and failure.

In addition to the examples listed in this paper, there exist many other cases where similar observations have been made regarding the damage evolution in recovered samples and an incomplete representation of damage by the PDV data [20]. Specifically, the anisotropic damage evolution when a material is shock loaded along the

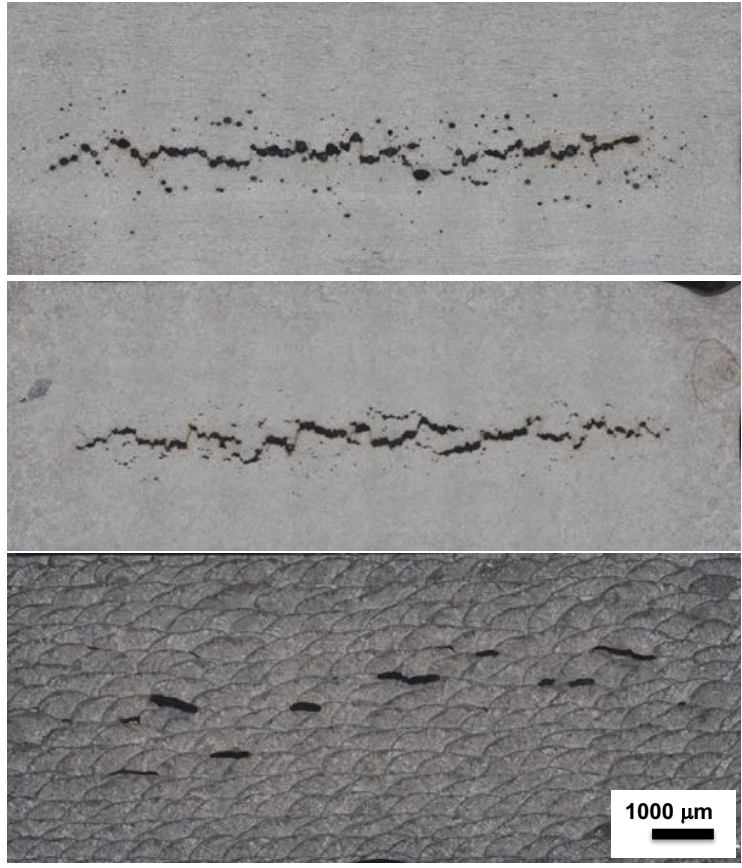

Fig. 2. Optical micrographs showing post-spall damage in 316L SS (top) wrought, (middle) AM heat treated and (bottom) AM. The larger black regions represent voids.

in-plane versus through-thickness directions is never well represented by the PDV data. This is clearly demonstrated in the case of $\mathrm{Zr}$ and $\mathrm{Ta}$ that were loaded along the in-plane and thru-thickness directions. Even though the PDV data was found to be nominally similar for loading along these two directions, the damage evolution was completely different [20]. This new criterion would be able to account for these differences in anisotropic evolution of damage. Similar observations exist for 1080 eutectoid steel and HY100 steel [20].

\section{Conclusions}

We have developed a new microstructure based criterion for failure that couples the spall strength with the total area of damage within a material. The importance of coupling the wave-profile signal with the damage evolution is to both assess the operative damage processes (such as ductile void formation, shear localization, brittle cracking, intergranular fracture) as well as the volume of the sample over which damage manifests. The development of microstructurally-aware predictive models for spallation is predicated on quantification of both the damage mechanism as well as the kinetics and volume within the sample over which this energy is dissipated. This new criterion is applied to a few problems reported in the literature to show that this criterion does indeed capture the resistance of material to total damage which includes void growth and coalescence and not just nucleation of voids. In the absence of microstructurally aware spall models, this criterion can be used as an indirect method of including microstructure into the damage models. This work is a step in 
the right direction and future work will involve addition of $3 \mathrm{D}$ volume effects obtained from X-ray Tomography type analysis into this new criterion.

\section{References}

1. G. I. Kanel, Spall Fracture: Methodological aspects, mechanisms and governing factors, Int. J. Frac. 163, 173-191 (2010)

2. D. Curran, L. Seaman, D. Shockley, Dynamic failure of solids, Phys. Reports, 147, 253 -388 (1987)

3. L. Seaman, D. R. Curran, D. A. Shockley, Computational models for ductile and brittle fracture, J. Appl. Phys. 47, 4814-26 (1976)

4. G. T. (Rusty) Gray III, High-strain-rate deformation: Mechanical behavior and deformation substructures induced, Ann. Rev. Mater. Res. 42, 285-303 (2012)

5. S. J. Fensin, E. K. walker, E. K. Cerreta, C. P. Trujillo, D. T. Martinez, and G. T. Gray III, Dynamic failure in two-phase materials, J. App. Phys. 18, 235305 (2015)

6. S. J. Fensin, E. K. Cerreta, G. T. Gray III and S. M. Valone, Why are some Interfaces in Materials Stronger than others, Sci. Rep. 4, 5641 (2014)

7. S. A. Novikov, I. I. Divnov. and A. G. Ivanov, The study of fracture of steel, aluminum, and copper under explosive loading, Phys. Met. Metallogr. 21, 122 (1966)

8. G.V. Stepanov, Spall fracture of metals by elasticplastic loading waves, Problemy Prochnosti 8, 66?70 (1976) (in Russian)

9. G. I. Kanel, S. V. Razorenov, K. Baumung, J. Singer, Dynamic yield and tensile strength of aluminum single crystals at temperatures up to the melting point, J Appl Phy 90, 136-143 (2001)
10. T. Antoun, L. Seaman, D. R. Curran,G. I. Kanel ,S. V. Razorenov, A. V. Utkin, Spall fracture, Springer, New York (2003)

11. M. A. Meyers, C. T. Aimone, Dynamic fracture (spalling) of metals, Prog. Mater. Sci 28, 1-96 (1983)

12. L. Seaman, D. Curran, D. Shockley, Dynamic failure in solids, Phys. Today 30, 46-55 (1977)

13. R. W. Minich, J. Cazamias, M. Kumar, A. Schwartz, Effect of microstructural length scales on spall behavior of copper, Metall. Mater. Trans. A 35, 2663-73 (2004)

14. R. W. Minich, M. Kumar, A. Schwatz, J. Cazamias, Scaling, microstructure and dynamic fracture, AIP Conf. Proc. 845, 642-45 (2006)

15. G. I. Kanel, S. V. Rasorenov, V. E. Fortov, Shock Wave and High-Strain-Rate Phenomena in Materials (Marcel Dekker, Cambridge, 1992)

16. G. T. Gray III, V. Livescu, P. A. Rigg, C. P. Trujillo, C. M. Cady, S. R. Chen, J. S. Carpenter, T. J. Lienert, S. J. Fensin, Structure/Property (constitutive and spallation response) of additively manufactured $316 \mathrm{~L}$ steel, Acta Mat. 138,140-149 (2017)

17. J. P. Escobedo-Diaz, D. Dennis-Koller, E. K. Cerreta, B. M. Patterson, C. A. Bronkhorst, B. L. Hansen, D. Tonks, R. A. Lebensohn, Effects of grain size and boundary structure on the dynamic tensile response of copper, J. Appl. Phys. 110, 033513 (2011)

18. W. D. Callister Jr, D. G. Rethwisch, Materials Science and Engineering, John Wiley and Sons, Hoboken, NJ (2008)

19. B. J. Jensen, D. B. Holtkamp, P. A. Rigg, and D. H. Dolan, J. Appl. Phys. 101, 013523 (2007)

20. G. T. Gray III, N. K. Bourne, K. S. Vecchio, J. C. F. Millett, Int J Frac 163, 243-258 (2010) 
